\title{
Exponential Stability for a Class of Uncertain Linear Systems with a Single Time-Delay (or Multiple Time-Delays)
}

\author{
Weiqiu Pan, Tianzeng $\mathrm{Li}^{*}$ \\ School of Mathematics and Statistics, Sichuan University of Science and Engineering, Zigong, China \\ Email: pwq237609@163.com, *litianzeng27@163.com
}

How to cite this paper: Pan, W.Q. and $\mathrm{Li}$, T.Z. (2021) Exponential Stability for a Class of Uncertain Linear Systems with a Single Time-Delay (or Multiple Time-Delays). Journal of Applied Mathematics and Physics, 9, 413-426.

https://doi.org/10.4236/jamp.2021.93029

Received: January 30, 2021

Accepted: March 14, 2021

Published: March 17, 2021

Copyright $\odot 2021$ by author(s) and Scientific Research Publishing Inc. This work is licensed under the Creative Commons Attribution International License (CC BY 4.0).

http://creativecommons.org/licenses/by/4.0/

\begin{abstract}
This paper investigates the issue of exponential stability for a class of uncertain linear systems with a single time-delay (or multiple time-delays). We consider that the uncertainties are the parameter disturbance and the external disturbance, both of which are stochastic. The external disturbances involve not only the current state $x(t)$ but also the delayed state $x(t-\tau)$. By means of the Lyapunov-Krasovskii functional, the sufficient conditions on exponential stability for the uncertain linear systems with a single time-delay (or multiple time-delays) are performed in the form of the linear matrix inequality (LMI). Selecting the suitable matrices $P$ (or $\tilde{P}$ ) and $Q$ (or $\tilde{Q}$ ) and parameter $\beta$ (or $\tilde{\beta}$ ), we can also get the bounds of the state variables for the single time-delay (or multiple time-delays) systems. In order to stabilize the solution of the single time-delay (or multiple time-delays) systems at the equilibrium point, we designed the state feedback control. Thus, the corresponding stabilization criteria are given. Finally, Numerical simulations show that a small disturbance can make a great change to the state variables of the systems. When the feedback gain control is added, the state variables of the systems can quickly stabilize at the equilibrium point. This also shows the effectiveness of the proposed method.
\end{abstract}

\section{Keywords}

Time-Delay System, Uncertainties, Lyapunov-Krasovskii Functional, The Linear Matrix Inequality (LMI)

\section{Introduction}

It's deemed important to analysis the stability of time-delay systems which has 
attracted widespread attention in the past decades. The investigation found that a system will deteriorate or even be unstable due to the interference of feedback delay, transmission delay and other factors. For example: due to the time lag, it may cause network congestion, communication data loss, traffic congestion in the transportation system, etc. Therefore, before designing a control system, it is necessary to explore the stability of the time-delay system, seeing survey papers [1] [2] [3] [4] [5]. From another level, in practical applications, the system will be affected by some factors such as environmental noise, or slowly changing parameters, and it is tough to obtain a stable mathematical model. We call these factors as uncertain factors, and their presence will also affect the stability of the system.

In literature [1], the author used the linear matrix inequality (LMI) method to give an exponential estimate and a sufficient condition for the generalized stability of linear time-delay systems. In 2009, Nam PHAN et al. [6] considered the linear polytopic time-delay systems under parameter disturbance, proposing the new sufficient conditions for the exponential stability and stabilization. And the controller they designed is only about the current state $x(t)$. Chen et al. [7] considered the nonlinear perturbations for the neutral-type time-delay systems. In terms of the linear matrix inequality (LMI), the new sufficient condition of stability with delay dependence is revealed. However, the nonlinear perturbations also only about the current state $x(t)$. Thus, in 2015, Tian et al. [8] researched the nonlinear perturbations which are about $x(t)$ and $x(t-\tau)$ in term of a class of time-varying delay systems. It can be better if the author considered the multiple time-delays systems. For the uncertain multiple time-delays systems, so far, there are few related studies. Literatures [9] [10] [11] [12] [13] exhibited the stabilities of multiple time-delays systems without any uncertainties. Chen et al. [14] focused on the sliding mode control for uncertain linear neutral systems with multiple delays. They just consider the parameter disturbance. Zhang et al. [15] investigated the robust stochastic stability for a class of uncertain stochastic systems with multiple delays adopting the method of multiple delays Lyapunov-Krasovskii function which the uncertainties were considered as a linear fractional form. Here are some methods for studying the stability of time-delay systems, such as the Laplace transform [9] [16], the Lyapunov direct method [17] [18], the linear matrix inequality used in this paper and so on. The Laplace transform method is too complicated to calculate and you need to construct a suitable Lyapunov function when you use the Lyapunov direct method which requires skill. In contrast, the linear matrix inequality method is simple, clear and is applicable to both integer order and fractional order systems.

Thus, we want to research the exponential stability for a class of linear single time-delay (or multiple time-delays) systems under parameter disturbance and external disturbance using the linear matrix inequality method. And the external disturbances are related not only with current state $x(t)$ but also with the de- 
layed state $x(t-\tau)$. Based on the definition of exponential stability, with the help of the Lyapunov-Krasovskii functional, we finally obtain the sufficient conditions of exponential stability for the uncertain linear systems with a single time-delay (or multiple time-delays) in the form of the linear matrix inequality (LMI). The state feedback control is designed to stabilize the uncertain linear time-delay systems. Numerical simulations show the effectiveness of our research method. The composition of this article is shown below. In chapter 2, we present the definitions, lemmas and the stabilization for the single time-delay system. In chapter 3, the stability for the multiple time-delays system is introduced. In chapter 4 , we have a sum up for this paper.

Notation: $\boldsymbol{I}_{n}$ represents the $n \times n$ identity matrix, $\mathbf{0}_{n}$ represents the $n \times n$ zero matrix.

\section{Preliminaries and the Stabilization of the Single Time-Delay System}

\subsection{Preliminaries}

Considering the uncertain time-delay system with controlled as follows

$$
\begin{aligned}
& \dot{\boldsymbol{x}}(t)=(\boldsymbol{A}+\Delta \boldsymbol{A}) \boldsymbol{x}(t)+(\boldsymbol{B}+\Delta \boldsymbol{B}) \boldsymbol{x}(t-\tau)+\boldsymbol{d}(x)+\boldsymbol{D u}(t) . \\
& \boldsymbol{x}(\theta)=\varphi(\theta), \quad \theta \in[-\tau, 0],
\end{aligned}
$$

where $\boldsymbol{x} \in \mathbb{R}^{n}$ is the state vector; $\boldsymbol{A}, \boldsymbol{B} \in \mathbb{R}^{n \times n}$ are the parameter matrices; $\Delta \boldsymbol{A}, \Delta \boldsymbol{B} \in \mathbb{R}^{n \times n}$ are the parameter interference matrices which satisfy $\|\Delta \boldsymbol{A}\| \leq m_{1}$ and $\|\Delta \boldsymbol{B}\| \leq m_{2} ; \boldsymbol{d}(\boldsymbol{x}, t)=\boldsymbol{G}_{1} \boldsymbol{x}(t)+\boldsymbol{G}_{2} \boldsymbol{x}(t-\tau)$ is external disturbances which $\boldsymbol{G}_{1}, \boldsymbol{G}_{2} \in \mathbb{R}^{n \times n} ; \tau \in \mathbb{R}^{+}$is the time delay; $\boldsymbol{D} \in \mathbb{R}^{n \times 1}$. We design the state feedback control as $\boldsymbol{u}(t)=\boldsymbol{E x}(t)$ which $\boldsymbol{E} \in \mathbb{R}^{1 \times n}$. For convenience, let $\boldsymbol{V}=\boldsymbol{D} \boldsymbol{E}$. The initial function defines as $\varphi \in \mathcal{C}\left([-\tau, 0], \mathbb{R}^{n}\right)$ with the uniform norm $\|\varphi\|_{\tau}=\max _{\varphi \in[-\tau, 0]}\|\varphi(\theta)\|$. It is stated that for any initial condition $\varphi \in \mathcal{C}\left([-\tau, 0], \mathbb{R}^{n}\right), t \geq 0$ there exists the unique solution $x(t, \varphi)$ for the system (1). We express $\boldsymbol{x}_{t}(\varphi)$ by the segment of trajectory $\boldsymbol{x}_{t}(\varphi)=\{\boldsymbol{x}(t+\theta, \varphi): \theta \in[-\tau, 0]\} \in \mathbb{R}^{n}$.

Definition 1 [19] Given $\xi>0$, the system (1) is considered to be exponentially stable if for every solution $x(t, \varphi)$ of the system, there exists a positive number $\sigma \geq 1$ such that meets

$$
\|x(t, \varphi)\| \leq \sigma \mathrm{e}^{\xi t}|\varphi|_{\tau}, \quad t \geq 0
$$

Lemma 1 Let $\boldsymbol{F}_{1}, \boldsymbol{F}_{2}, \boldsymbol{W}_{1}, \boldsymbol{W}_{2}, \boldsymbol{H} \in \mathbb{R}^{n \times n}$ be matrices such that $\left\|\boldsymbol{F}_{1}\right\| \leq \delta_{1}$ and $\left\|\boldsymbol{F}_{2}\right\| \leq \delta_{2}, \quad \boldsymbol{u}, \boldsymbol{v} \in \mathbb{R}^{n \times 1}$ be the vector. Then, the following majorization holds:

$$
\begin{aligned}
& {\left[\begin{array}{l}
\boldsymbol{u} \\
\boldsymbol{v}
\end{array}\right]^{\mathrm{T}}\left[\begin{array}{l}
\boldsymbol{F}_{1} \\
\boldsymbol{F}_{2}
\end{array}\right] \boldsymbol{H}\left[\begin{array}{ll}
\boldsymbol{W}_{1} & \boldsymbol{W}_{2}
\end{array}\right]\left[\begin{array}{l}
\boldsymbol{u} \\
\boldsymbol{v}
\end{array}\right]} \\
& \leq \rho \sqrt{\delta_{1}^{2}+\delta_{2}^{2}} \sqrt{\left\|\boldsymbol{W}_{1} \boldsymbol{W}_{1}^{\mathrm{T}}+\boldsymbol{W}_{2} \boldsymbol{W}_{2}^{\mathrm{T}}\right\|}\left[\begin{array}{l}
\boldsymbol{u} \\
\boldsymbol{v}
\end{array}\right]^{\mathrm{T}}\left[\begin{array}{ll}
\boldsymbol{I}_{n} & \mathbf{0}_{n} \\
\mathbf{0}_{n} & \boldsymbol{I}_{n}
\end{array}\right]\left[\begin{array}{l}
\boldsymbol{u} \\
\boldsymbol{v}
\end{array}\right]
\end{aligned}
$$

for satisfying

$$
\boldsymbol{H}<\lambda_{\max }(\boldsymbol{H}) \boldsymbol{I}_{n}=\rho \boldsymbol{I}_{n}
$$


Proof. You can refer to [[1], Appendix, Lemma 2] for the process of proof.

Lemma 2 Let $\boldsymbol{F}=\left[\boldsymbol{F}_{1}, \boldsymbol{F}_{2}, \cdots, \boldsymbol{F}_{m}\right]^{\mathrm{T}} \in \mathbb{R}^{n m \times n}, \quad \boldsymbol{W}=\left[\boldsymbol{W}_{1}, \boldsymbol{W}_{2}, \cdots, \boldsymbol{W}_{m}\right] \in \mathbb{R}^{n \times n m}$ with $\boldsymbol{F}_{i}, \boldsymbol{W}_{i}, \boldsymbol{H} \in \mathbb{R}^{n \times n}$ be matrices such that $\left\|\boldsymbol{F}_{i}\right\| \leq \delta_{\boldsymbol{F}_{i}}, i=(1,2, \cdots, m), \quad \boldsymbol{u} \in \mathbb{R}^{n \times 1}$, and $\boldsymbol{v} \in \mathbb{R}^{n(m-1) \times 1}$ be the vector. Then, the following majorization holds:

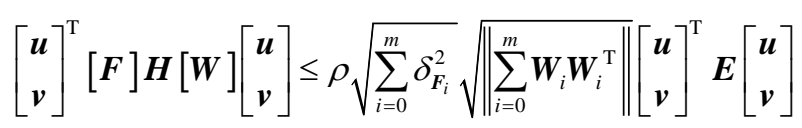

for satisfying

$$
\boldsymbol{H}<\lambda_{\max }(\boldsymbol{H}) \boldsymbol{I}_{n}=\rho \boldsymbol{I}_{n},
$$

where $\boldsymbol{E}=\operatorname{diag}[\underbrace{\boldsymbol{I}_{n}, \boldsymbol{I}_{n}, \cdots, \boldsymbol{I}_{n}}_{m}]$.

Proof. In [[1], Appendix, Lemma 2], the author considered the two matrices while we consider the number of matrices is $m$.

\subsection{The Stabilization of the Single Time-Delay System}

Theorem 1 The controlled system (1) is exponential stability if there exist two real positive matrices $\boldsymbol{P}, \boldsymbol{Q} \in \mathbb{R}^{n \times n}$ and the constant $\beta>0$ and $\rho>0$ such that the conditions

$$
\begin{aligned}
& \mathcal{M}(\boldsymbol{P}, \boldsymbol{Q})+\rho \boldsymbol{K}\left(m_{1}, m_{2}\right)+2 \beta \mathcal{M}(\boldsymbol{P})<0 \\
& \boldsymbol{P}<\rho \boldsymbol{I}
\end{aligned}
$$

hold. Then

$$
\|\boldsymbol{x}(t, \varphi)\| \leq \sqrt{\frac{\alpha_{2}}{\alpha_{1}}} \mathrm{e}^{-\beta t}\|\varphi\|_{\tau}
$$

where

$$
\begin{gathered}
\rho=\lambda_{\text {max }}(\boldsymbol{P}), \quad \alpha_{1}=\lambda_{\min }(\boldsymbol{P}), \quad \alpha_{2}=\rho+\tau \lambda_{\max }(\boldsymbol{Q}), \\
\boldsymbol{K}\left(m_{1}, m_{2}\right)=2 \sqrt{m_{1}^{2}+m_{2}^{2}}\left[\begin{array}{ll}
\boldsymbol{I}_{n} & \mathbf{0}_{n} \\
\mathbf{0}_{n} & \boldsymbol{I}_{n}
\end{array}\right], \\
\mathcal{M}(\boldsymbol{P}, \boldsymbol{Q})=\left[\begin{array}{cc}
* & \boldsymbol{P} \boldsymbol{B}+\boldsymbol{P} \boldsymbol{G}_{2} \\
\boldsymbol{B}^{\mathrm{T}} \boldsymbol{P}+\boldsymbol{G}_{2}^{\mathrm{T}} \boldsymbol{P} & -\mathrm{e}^{-2 \beta \tau} \boldsymbol{Q}
\end{array}\right], \\
\mathcal{M}(\boldsymbol{P})=\left[\begin{array}{c}
\boldsymbol{I}_{n} \\
\mathbf{0}_{n}
\end{array}\right] \boldsymbol{P}\left[\begin{array}{ll}
\boldsymbol{I}_{n} & \mathbf{0}_{n}
\end{array}\right]=\left[\begin{array}{cc}
\boldsymbol{P} & \mathbf{0}_{n} \\
\mathbf{0}_{n} & \mathbf{0}_{n}
\end{array}\right] .
\end{gathered}
$$

with $*=\boldsymbol{P A}+\boldsymbol{A}^{\mathrm{T}} \boldsymbol{P}+\boldsymbol{Q}+\boldsymbol{G}_{1}+\boldsymbol{G}_{1}^{\mathrm{T}}+\boldsymbol{V}^{\mathrm{T}}+\boldsymbol{V}$.

Proof. We present the Lyapunov-Krasovskii functional

$$
V\left(\boldsymbol{x}_{t}\right)=\boldsymbol{x}(t)^{\mathrm{T}} \boldsymbol{P} \boldsymbol{x}(t)+\int_{-\tau}^{0} \boldsymbol{x}(t+\theta)^{\mathrm{T}} \mathrm{e}^{2 \beta \theta} \boldsymbol{Q} \boldsymbol{x}(t+\theta) \mathrm{d} \theta .
$$

Because $\int_{-\tau}^{0} \boldsymbol{x}(t+\theta)^{\mathrm{T}} \mathrm{e}^{2 \beta \theta} \boldsymbol{Q} \boldsymbol{x}(t+\theta) \mathrm{d} \theta=\left\|\boldsymbol{x}_{t}\right\|_{\tau}^{2} \boldsymbol{Q} \int_{-\tau}^{0} \mathrm{e}^{2 \beta \theta} \mathrm{d} \theta \leq \tau\left\|\boldsymbol{x}_{t}\right\|_{\tau}^{2} \boldsymbol{Q}$. From (7), the following inequalities can be obtained.

$$
\alpha_{1}\|\boldsymbol{x}(t)\|^{2} \leq V\left(\boldsymbol{x}_{t}\right) \leq \alpha_{2}\left\|\boldsymbol{x}_{t}\right\|_{\tau}^{2},
$$

where $\alpha_{1}, \alpha_{2}$ are defined in Theorem 1 . 
Observe that $V\left(x_{t}\right)$ can be rewritten as

$$
V\left(\boldsymbol{x}_{t}\right)=\left(\begin{array}{c}
\boldsymbol{x}(t) \\
\boldsymbol{x}(t-\tau)
\end{array}\right)^{\mathrm{T}} \mathcal{M}(\boldsymbol{P})\left(\begin{array}{c}
\boldsymbol{x}(t) \\
\boldsymbol{x}(t-\tau)
\end{array}\right)+\int_{-\tau}^{0} \boldsymbol{x}(t+\theta)^{\mathrm{T}} \mathrm{e}^{2 \beta \theta} \boldsymbol{Q} \boldsymbol{x}(t+\theta) \mathrm{d} \theta
$$

where $\mathcal{M}(\boldsymbol{P})$ is defined in Theorem 1 .

The derivative of the functional $V\left(x_{t}\right)$ along the trajectories of the uncertain system (1) is

$$
\begin{aligned}
\frac{\mathrm{d}}{\mathrm{d} t} V\left(\boldsymbol{x}_{t}\right)= & 2 \boldsymbol{x}^{\mathrm{T}}(t) \boldsymbol{P}\{(\boldsymbol{A}+\Delta \boldsymbol{A}) \boldsymbol{x}(t)+(\boldsymbol{B}+\Delta \boldsymbol{B}) \boldsymbol{x}(t-\tau)+\boldsymbol{d}(x)+\boldsymbol{D} \boldsymbol{u}(t)\} \\
& +\boldsymbol{x}^{\mathrm{T}}(t) \boldsymbol{Q} \boldsymbol{x}(t)-\boldsymbol{x}^{\mathrm{T}}(t-\tau) \mathrm{e}^{-2 \beta \tau} \boldsymbol{Q} \boldsymbol{x}(t-\tau) \\
& -2 \beta \int_{-\tau}^{0} \boldsymbol{x}(t+\theta)^{\mathrm{T}} \mathrm{e}^{2 \beta \theta} \boldsymbol{Q} \boldsymbol{x}(t+\theta) \mathrm{d} \theta .
\end{aligned}
$$

It can be rewritten as

$$
\begin{aligned}
\frac{\mathrm{d}}{\mathrm{d} t} V\left(\boldsymbol{x}_{t}\right)= & {\left[\begin{array}{c}
\boldsymbol{x}(t) \\
\boldsymbol{x}(t-\tau)
\end{array}\right]^{\mathrm{T}}\left\{\mathcal{M}(\boldsymbol{P}, \boldsymbol{Q})+2\left[\begin{array}{c}
(\Delta \boldsymbol{A})^{\mathrm{T}} \\
(\Delta \boldsymbol{B})^{\mathrm{T}}
\end{array}\right] \boldsymbol{P}\left[\begin{array}{ll}
\boldsymbol{I}_{n} & \mathbf{0}_{n}
\end{array}\right]\right\}\left[\begin{array}{c}
\boldsymbol{x}(t) \\
\boldsymbol{x}(t-\tau)
\end{array}\right] } \\
& -2 \beta \int_{-\tau}^{0} \boldsymbol{x}(t+\theta)^{\mathrm{T}} \mathrm{e}^{2 \beta \theta} \boldsymbol{Q} \boldsymbol{x}(t+\theta) \mathrm{d} \theta,
\end{aligned}
$$

where $\mathcal{M}(\boldsymbol{P}, \boldsymbol{Q})$ is defined in Theorem 1 .

It follows from Lemmal that the following result is hold.

$$
\begin{aligned}
\frac{\mathrm{d}}{\mathrm{d} t} V\left(\boldsymbol{x}_{t}\right) \leq & {\left[\begin{array}{c}
\boldsymbol{x}(t) \\
\boldsymbol{x}(t-\tau)
\end{array}\right]^{\mathrm{T}}\left\{\mathcal{M}(\boldsymbol{P}, \boldsymbol{Q})+\rho \boldsymbol{K}\left(m_{1}, m_{2}\right)\right\}\left[\begin{array}{c}
\boldsymbol{x}(t) \\
\boldsymbol{x}(t-\tau)
\end{array}\right] } \\
& -2 \beta \int_{-\tau}^{0} \boldsymbol{x}(t+\theta)^{\mathrm{T}} \mathrm{e}^{2 \beta \theta} \boldsymbol{Q} \boldsymbol{x}(t+\theta) \mathrm{d} \theta
\end{aligned}
$$

where

$$
\boldsymbol{K}\left(m_{1}, m_{2}\right)=2 \sqrt{m_{1}^{2}+m_{2}^{2}}\left[\begin{array}{cc}
\boldsymbol{I}_{n} & \mathbf{0}_{n} \\
\mathbf{0}_{n} & \boldsymbol{I}_{n}
\end{array}\right]
$$

Finally, we get

$$
\begin{aligned}
& \frac{\mathrm{d}}{\mathrm{d} t} V\left(\boldsymbol{x}_{t}\right)+2 \beta V\left(\boldsymbol{x}_{t}\right) \\
& \leq\left[\begin{array}{c}
\boldsymbol{x}(t) \\
\boldsymbol{x}(t-\tau)
\end{array}\right]^{\mathrm{T}}\left\{\mathcal{M}(\boldsymbol{P}, \boldsymbol{Q})+\rho \boldsymbol{K}\left(m_{1}, m_{2}\right)+2 \beta \mathcal{M}(\boldsymbol{P})\right\}\left[\begin{array}{c}
\boldsymbol{x}(t) \\
\boldsymbol{x}(t-\tau)
\end{array}\right] .
\end{aligned}
$$

When the matrix $\mathcal{M}(\boldsymbol{P}, \boldsymbol{Q})+\rho \boldsymbol{K}\left(m_{1}, m_{2}\right)+2 \beta \mathcal{M}(\boldsymbol{P})$ is a negative matrix, we can obtain

$$
\frac{\mathrm{d}}{\mathrm{d} t} V\left(\boldsymbol{x}_{t}(\varphi)\right)+2 \beta V\left(\boldsymbol{x}_{t}(\varphi)\right) \leq 0
$$

This inequality bring about the following one

$$
V\left(\boldsymbol{x}_{t}(\varphi)\right) \leq \mathrm{e}^{-2 \beta t} V(\varphi), \text { for } t \geq 0
$$

Thus, it follows from the Equation (8) that

$$
\alpha_{1}\|\boldsymbol{x}(t, \varphi)\|^{2} \leq V\left(x_{t}(\varphi)\right) \leq \mathrm{e}^{-2 \beta t} V(\varphi) \leq \alpha_{2} \mathrm{e}^{-2 \beta t}\|\varphi\|_{\tau}^{2} \text { for } t \geq 0 .
$$


Then, $\|x(t, \varphi)\| \leq \sqrt{\frac{\alpha_{2}}{\alpha_{1}}} \mathrm{e}^{-\beta t}\|\varphi\|_{\tau}, t \geq 0$.

Example 1 These matrixes in Theorem 1 are chose as follows

$$
\begin{gathered}
\boldsymbol{P}=\left[\begin{array}{ll}
8 & 2 \\
2 & 5
\end{array}\right], \quad \boldsymbol{Q}=\left[\begin{array}{cc}
9.8 & 8 \\
8 & 9
\end{array}\right], \quad \boldsymbol{A}=\left[\begin{array}{cc}
-2.5 & 0.3 \\
0 & -1
\end{array}\right], \quad \boldsymbol{B}=\left[\begin{array}{cc}
0.2 & 0.6 \\
0 & 0.4
\end{array}\right], \\
\boldsymbol{G}_{1}=\left[\begin{array}{ll}
0.2 & 0.3 \\
0.1 & 0.2
\end{array}\right], \quad \boldsymbol{G}_{2}=\left[\begin{array}{ll}
0.2 & 0.4 \\
0.1 & 0.3
\end{array}\right], \quad \boldsymbol{V}=\boldsymbol{D} \boldsymbol{E}=\left[\begin{array}{c}
4 \\
10
\end{array}\right] *\left[\begin{array}{ll}
0.02 & -2.7
\end{array}\right], \\
\Delta \boldsymbol{A}=\left[\begin{array}{cc}
0.1 \sin (t) & 0.1 \sin (t) \\
0.1 \sin (2 t) & 0.1 \sin (3 t)
\end{array}\right], \quad \Delta \boldsymbol{B}=\left[\begin{array}{cc}
0.1 \cos (t) & 0.1 \cos (t) \\
0.1 \cos (2 t) & 0.1 \cos (3 t)
\end{array}\right],
\end{gathered}
$$

We choose $\Delta \boldsymbol{A} \leq 0.2, \Delta \boldsymbol{B} \leq 0.2, \tau=0.5, \quad \beta=0.5$. By calculating, we obtained

$$
\begin{aligned}
& \mathcal{M}(\boldsymbol{P}, \boldsymbol{Q})+\rho \boldsymbol{K}\left(m_{1}, m_{2}\right)+2 \beta \mathcal{M}(\boldsymbol{P}) \\
& =\left[\begin{array}{cccc}
-16.5488 & 0.2912 & 3.4000 & 9.4000 \\
0.2912 & -43.3088 & 1.3000 & 5.5000 \\
3.4000 & 1.3000 & -11.0663 & -8.0986 \\
9.4000 & 5.5000 & -8.0986 & -9.7473
\end{array}\right],
\end{aligned}
$$

which the eigenvalues are

$$
\lambda_{1}=-0.1236, \lambda_{2}=-9.9004, \lambda_{3}=-26.1449, \lambda_{4}=-44.5025 .
$$

Thus, the matrix $\mathcal{M}(\boldsymbol{P}, \boldsymbol{Q})+\rho \boldsymbol{K}\left(m_{1}, m_{2}\right)+2 \beta \mathcal{M}(\boldsymbol{P})$ ia a negative matrix and $\boldsymbol{P}<\rho \boldsymbol{I}$. The two conditions in Theorem 2 are satisfied. At this time, the solution of the system meets

$$
\|x(t, \varphi)\| \leq 2.1039 \mathrm{e}^{-0.5 t}\|\varphi\|_{\tau} .
$$

When we select the initial values as $\varphi(\theta)=(-2,2)^{\mathrm{T}}, t \in[-3,0]$. The trajectories of the state variables $x_{1}(t)$ and $x_{2}(t)$ for the uncertain time-delay system without the controller are depicted in Figure 1 while the Figure 2 expresses the trajectories of the state variables $x_{1}(t)$ and $x_{2}(t)$ for the controlled uncertain time-delay system (1). Numerical simulation results reveal the effectiveness of our proposed method.

\section{The Stabilization of the Multiple Time-Delays Syetem}

\subsection{The Stability of the Multiple Time-Delays System}

We are concentrating on the case of the uncertain system with multiple time-delays in the following form

$$
\begin{aligned}
& \dot{\boldsymbol{x}}(t)=(\boldsymbol{A}+\Delta \boldsymbol{A}) \boldsymbol{x}(t)+\sum_{i=1}^{m}\left(\boldsymbol{B}_{i}+\Delta \boldsymbol{B}_{i}\right) \boldsymbol{x}\left(t-\tau_{i}\right)+\boldsymbol{d}(t) \\
& \boldsymbol{x}(\theta)=\varphi(\theta), \quad \theta \in[-\tau, 0]
\end{aligned}
$$

where $\boldsymbol{B}_{i} \in \mathbb{R}^{n \times n},(i=1, \cdots, m)$ and $0<\tau_{1}, \cdots, \tau_{m}<\tau$ are delays. The parameter interference matrices $\Delta \boldsymbol{A}, \Delta \boldsymbol{B}_{i}$ satisfy $\|\Delta \boldsymbol{A}\| \leq \omega$ and $\left\|\Delta \boldsymbol{B}_{i}\right\| \leq v_{i},(i=1, \cdots, m)$; $\boldsymbol{d}(\boldsymbol{x}, t)=\tilde{\boldsymbol{G}}_{0} \boldsymbol{x}(t)+\sum_{i=1}^{m} \tilde{\boldsymbol{G}}_{i} \boldsymbol{x}\left(t-\tau_{i}\right)$ is external disturbances. 


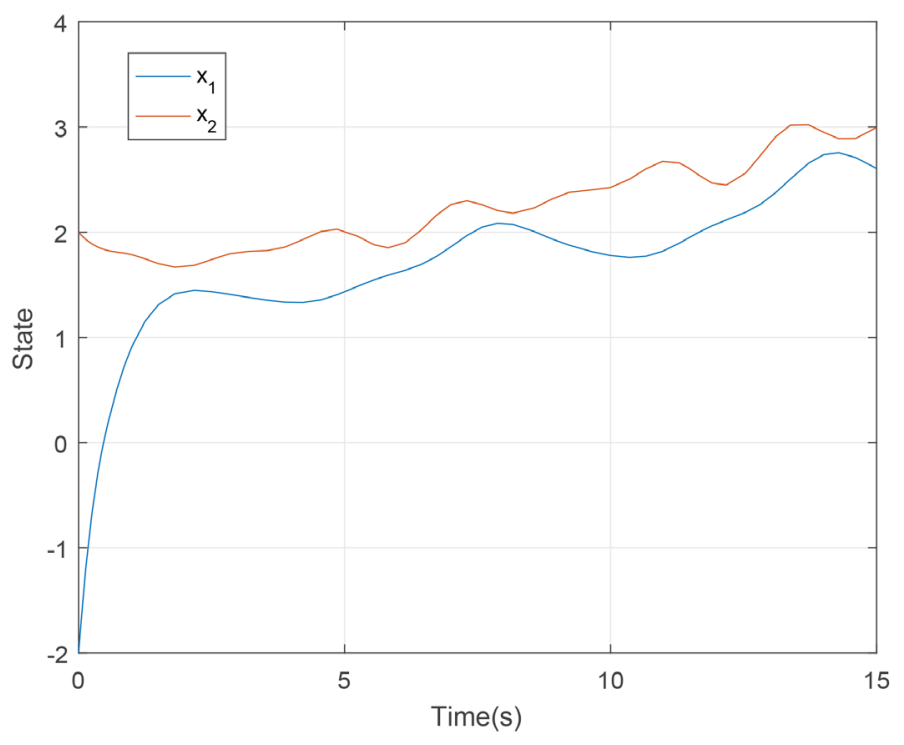

Figure 1. The trajectories of the state variables $x_{1}(t)$ and $x_{2}(t)$ for the uncertain single time-delay system without the controller.

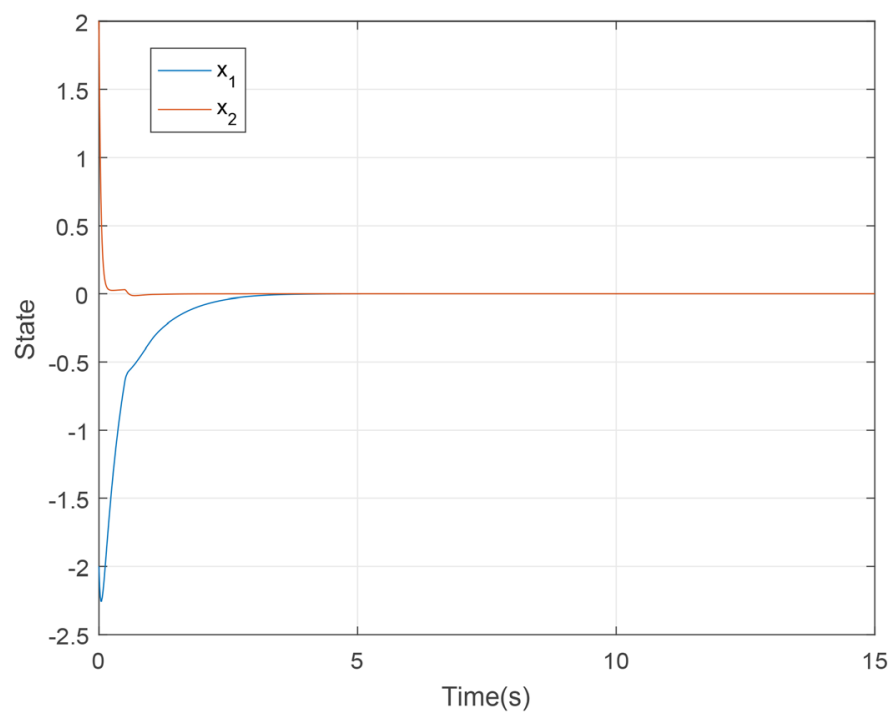

Figure 2. The trajectories of the state variables $x_{1}(t)$ and $x_{2}(t)$ for the uncertain single time-delay system with controlled (1).

Theorem 2 The uncertain multiple time-delays system (19) is exponential stability if there exist real positive matrices $\tilde{\boldsymbol{P}}, \tilde{\boldsymbol{Q}}_{i},(i=1,2, \cdots, m) \in \mathbb{R}^{n \times n}$ and the constant $\tilde{\beta}>0$ and $\tilde{\rho}>0$ such that the conditions

$$
\begin{aligned}
& \tilde{\mathcal{M}}\left(\tilde{\boldsymbol{P}}, \tilde{\boldsymbol{Q}}_{1}, \tilde{\boldsymbol{Q}}_{2}, \cdots, \tilde{\boldsymbol{Q}}_{m}\right)+\tilde{\rho} \boldsymbol{K}(\omega, \boldsymbol{u})+2 \tilde{\beta} \tilde{\mathcal{M}}(\tilde{\boldsymbol{P}})<0 \\
& \tilde{\boldsymbol{P}}<\tilde{\rho} \mathbf{I}
\end{aligned}
$$

hold. Then

$$
\|\boldsymbol{x}(t, \varphi)\| \leq \sqrt{\frac{\tilde{\alpha}_{2}}{\tilde{\alpha}_{1}}} \mathrm{e}^{-\tilde{\beta} t}\|\varphi\|_{\tau}
$$


where

$$
\begin{aligned}
& \tilde{\rho}=\lambda_{\max }(\tilde{\boldsymbol{P}}), \quad \tilde{\alpha}_{1}=\lambda_{\min }(\tilde{\boldsymbol{P}}), \quad \tilde{\alpha}_{2}=\tilde{\rho}+\sum_{i=1}^{m} \tau_{i} \lambda_{\max }\left(\tilde{\boldsymbol{Q}}_{i}\right), \\
& \boldsymbol{K}(\omega, \boldsymbol{u})=2 \sqrt{\omega^{2}+\sum_{i=1}^{m} v_{i}^{2}} \boldsymbol{E}, \\
& \tilde{\mathcal{M}}\left(\tilde{\boldsymbol{P}}, \tilde{\boldsymbol{Q}}_{1}, \tilde{\boldsymbol{Q}}_{2}, \cdots, \tilde{\boldsymbol{Q}}_{m}\right) \\
& =\operatorname{diag}\left(\tilde{\boldsymbol{P}} \boldsymbol{A}+\boldsymbol{A}^{\mathrm{T}} \tilde{\boldsymbol{P}}+\sum_{i=1}^{m} \tilde{\boldsymbol{Q}}_{i}+\tilde{\boldsymbol{G}}_{0}+\tilde{\boldsymbol{G}}_{0}^{\mathrm{T}},-\mathrm{e}^{-2 \tilde{\beta} \tau_{1}} \tilde{\boldsymbol{Q}}_{1}, \cdots,-\mathrm{e}^{-2 \tilde{\tilde{\tau}} \tau_{m}} \tilde{\boldsymbol{Q}}_{m}\right) \\
& +\left(\boldsymbol{B}^{T}+\boldsymbol{G}^{\mathrm{T}}\right) \tilde{\boldsymbol{P}} \boldsymbol{E}_{*}+\boldsymbol{E}_{*}^{\mathrm{T}} \tilde{\boldsymbol{P}}(\boldsymbol{B}+\boldsymbol{G}),
\end{aligned}
$$

with

$$
\begin{gathered}
\boldsymbol{B}=\left[\boldsymbol{B}_{1}, \boldsymbol{B}_{2}, \cdots, \boldsymbol{B}_{m}\right], \quad \boldsymbol{G}=\left[\tilde{\boldsymbol{G}}_{1}, \tilde{\boldsymbol{G}}_{2}, \cdots, \tilde{\boldsymbol{G}}_{m}\right], \quad \boldsymbol{E}_{*}=[\boldsymbol{I}_{n}, \underbrace{\mathbf{0}_{n}, \cdots, \mathbf{0}_{n}}_{m-1}], \\
\boldsymbol{E}=\operatorname{diag}[\underbrace{\boldsymbol{I}_{n}, \boldsymbol{I}_{n}, \cdots, \boldsymbol{I}_{n}}_{m}], \quad \tilde{\mathcal{M}}(\tilde{\boldsymbol{P}})=\operatorname{diag}\{\underbrace{\tilde{\boldsymbol{P}}, \underbrace{}_{n}, \cdots, \mathbf{0}_{n}}_{m}\} .
\end{gathered}
$$

Proof. We introduce the Lyapunov-Krasovskii functional

$$
\tilde{V}\left(\boldsymbol{x}_{t}\right)=\boldsymbol{x}(t)^{\mathrm{T}} \tilde{\boldsymbol{P}} \boldsymbol{x}(t)+\sum_{i=1}^{m} \int_{-\tau_{i}}^{0} \boldsymbol{x}(t+\theta)^{\mathrm{T}} \mathrm{e}^{2 \tilde{\beta} \theta} \tilde{\boldsymbol{Q}}_{i} \boldsymbol{x}(t+\theta) \mathrm{d} \theta .
$$

Notice that $\tilde{V}\left(\boldsymbol{x}_{t}\right)$ can be expressed as

$$
\begin{aligned}
\tilde{V}\left(\boldsymbol{x}_{t}\right)= & {\left[\begin{array}{c}
\boldsymbol{x}(t) \\
\boldsymbol{x}\left(t-\tau_{1}\right) \\
\boldsymbol{x}\left(t-\tau_{2}\right) \\
\vdots \\
\boldsymbol{x}\left(t-\tau_{m}\right)
\end{array}\right]^{\mathrm{T}} \tilde{\mathcal{M}}(\tilde{\boldsymbol{P}})\left[\begin{array}{c}
\boldsymbol{x}(t) \\
\boldsymbol{x}\left(t-\tau_{1}\right) \\
\boldsymbol{x}\left(t-\tau_{2}\right) \\
\vdots \\
\boldsymbol{x}\left(t-\tau_{m}\right)
\end{array}\right] } \\
& +\sum_{i=1}^{m} \int_{-\tau_{i}}^{0} \boldsymbol{x}(t+\theta)^{\mathrm{T}} \mathrm{e}^{2 \tilde{\beta} \theta} \tilde{\boldsymbol{Q}}_{i} \boldsymbol{x}(t+\theta) \mathrm{d} \theta,
\end{aligned}
$$

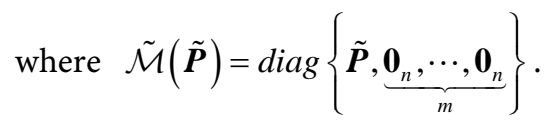

The derivative of the functional $V\left(\boldsymbol{x}_{t}\right)$ along the trajectories of the uncertain system (19) is

$$
\begin{aligned}
\frac{\mathrm{d}}{\mathrm{d} t} \tilde{V}\left(\boldsymbol{x}_{t}\right)= & 2 \boldsymbol{x}^{\mathrm{T}}(t) \tilde{\boldsymbol{P}}\left\{(\boldsymbol{A}+\Delta \boldsymbol{A}) \boldsymbol{x}(t)+\sum_{i=1}^{m}\left(\boldsymbol{B}_{i}+\Delta \boldsymbol{B}_{i}\right) \boldsymbol{x}\left(t-\tau_{i}\right)+\boldsymbol{d}(t)\right\} \\
& +\sum_{i=1}^{m} \boldsymbol{x}^{\mathrm{T}}(t) \boldsymbol{Q} \boldsymbol{x}(t)-\sum_{i=1}^{m} \boldsymbol{x}\left(t-\tau_{i}\right)^{\mathrm{T}} \mathrm{e}^{-2 \tilde{\beta} \tau_{i}} \tilde{\boldsymbol{Q}} \boldsymbol{x}\left(t-\tau_{i}\right) \\
& -2 \tilde{\beta} \sum_{i=1}^{m} \int_{-\tau_{i}}^{0} \boldsymbol{x}(t+\theta)^{\mathrm{T}} \mathrm{e}^{2 \tilde{\beta} \theta} \tilde{\boldsymbol{Q}}_{i} \boldsymbol{x}(t+\theta) \mathrm{d} \theta .
\end{aligned}
$$

It can be rewritten as 


$$
\begin{aligned}
\frac{\mathrm{d}}{\mathrm{d} t} \tilde{V}\left(\boldsymbol{x}_{t}\right)= & {\left[\begin{array}{c}
\boldsymbol{x}(t) \\
\boldsymbol{x}\left(t-\tau_{1}\right) \\
\boldsymbol{x}\left(t-\tau_{2}\right) \\
\vdots \\
\boldsymbol{x}\left(t-\tau_{m}\right)
\end{array}\right]^{\mathrm{T}}\left\{\tilde{\mathcal{M}}\left(\tilde{\boldsymbol{P}}, \tilde{\boldsymbol{Q}}_{1}, \tilde{\boldsymbol{Q}_{2}}, \cdots, \tilde{\boldsymbol{Q}}_{m}\right)+2\left[\begin{array}{c}
(\Delta A)^{\mathrm{T}} \\
\left(\Delta B_{1}\right)^{\mathrm{T}} \\
\vdots \\
\left(\Delta B_{m}\right)^{\mathrm{T}}
\end{array}\right] \boldsymbol{P E}\right\} } \\
& \times\left[\begin{array}{c}
\boldsymbol{x}(t) \\
\boldsymbol{x}\left(t-\tau_{1}\right) \\
\boldsymbol{x}\left(t-\tau_{2}\right) \\
\vdots \\
\boldsymbol{x}\left(t-\tau_{m}\right)
\end{array}\right]-2 \tilde{\beta} \sum_{i=1}^{m} \int_{-\tau_{i}}^{0} \boldsymbol{x}(t+\theta)^{\mathrm{T}} \mathrm{e}^{2 \tilde{\beta} \theta} \tilde{\boldsymbol{Q}}_{i} \boldsymbol{x}(t+\theta) \mathrm{d} \theta,
\end{aligned}
$$

where $\tilde{\mathcal{M}}\left(\tilde{\boldsymbol{P}}, \tilde{\boldsymbol{Q}}_{1}, \tilde{\boldsymbol{Q}}_{2}, \cdots, \tilde{\boldsymbol{Q}}_{m}\right)$ and $\boldsymbol{E}$ are defined in Theorem 2 .

It follows from Lemma 2 that the following result is hold.

$$
\begin{aligned}
\frac{\mathrm{d}}{\mathrm{d} t} \tilde{V}\left(\boldsymbol{x}_{t}\right) \leq & {\left[\begin{array}{c}
\boldsymbol{x}(t) \\
\boldsymbol{x}\left(t-\tau_{1}\right) \\
\boldsymbol{x}\left(t-\tau_{2}\right) \\
\vdots \\
\boldsymbol{x}\left(t-\tau_{m}\right)
\end{array}\right]^{\mathrm{T}}\left\{\tilde{\mathcal{M}}\left(\tilde{\boldsymbol{P}}, \tilde{\boldsymbol{Q}}_{1}, \tilde{\boldsymbol{Q}}_{2}, \cdots, \tilde{\boldsymbol{Q}}_{m}\right)+\tilde{\rho} \boldsymbol{K}(\omega, \boldsymbol{u})\right\}\left[\begin{array}{c}
\boldsymbol{x}(t) \\
\boldsymbol{x}\left(t-\tau_{1}\right) \\
\boldsymbol{x}\left(t-\tau_{2}\right) \\
\vdots \\
\boldsymbol{x}\left(t-\tau_{m}\right)
\end{array}\right] } \\
& -2 \tilde{\beta} \sum_{i=1}^{m} \int_{-\tau_{i}}^{0} \boldsymbol{x}^{\mathrm{T}}(t+\theta) \mathrm{e}^{2 \tilde{\beta} \theta} \tilde{\boldsymbol{Q}}_{i} \boldsymbol{x}(t+\theta) \mathrm{d} \theta,
\end{aligned}
$$

where

$$
\boldsymbol{K}(\omega, \boldsymbol{u})=2 \sqrt{\omega^{2}+\sum_{i=0}^{m} v_{i}^{2}} \boldsymbol{E}
$$

Finally, we get

$$
\begin{aligned}
& \frac{\mathrm{d}}{\mathrm{d} t} \tilde{V}\left(\boldsymbol{x}_{t}\right)+2 \tilde{\beta} \tilde{V}\left(\boldsymbol{x}_{t}\right) \\
& \leq\left[\begin{array}{c}
\boldsymbol{x}(t) \\
\boldsymbol{x}\left(t-\tau_{1}\right) \\
\boldsymbol{x}\left(t-\tau_{2}\right) \\
\vdots \\
\boldsymbol{x}\left(t-\tau_{m}\right)
\end{array}\right]^{\mathrm{T}}\left\{\tilde{\mathcal{M}}\left(\tilde{\boldsymbol{P}}, \tilde{\boldsymbol{Q}}_{1}, \tilde{\boldsymbol{Q}}_{2}, \cdots, \tilde{\boldsymbol{Q}}_{m}\right)+\tilde{\rho} \boldsymbol{K}(\omega, \boldsymbol{u})+2 \tilde{\beta} \tilde{\mathcal{M}}(\tilde{\boldsymbol{P}})\right\}\left[\begin{array}{c}
\boldsymbol{x}(t) \\
\boldsymbol{x}\left(t-\tau_{1}\right) \\
\boldsymbol{x}\left(t-\tau_{2}\right) \\
\vdots \\
\boldsymbol{x}\left(t-\tau_{m}\right)
\end{array}\right] .
\end{aligned}
$$

When the matrix $\tilde{\mathcal{M}}\left(\tilde{\boldsymbol{P}}, \tilde{\boldsymbol{Q}}_{1}, \tilde{\boldsymbol{Q}}_{2}, \cdots, \tilde{\boldsymbol{Q}}_{m}\right)+\tilde{\rho} \boldsymbol{K}(\omega, \boldsymbol{u})+2 \tilde{\beta} \tilde{\mathcal{M}}(\tilde{\boldsymbol{P}})$ is a negative matrix, we can obtain

$$
\frac{\mathrm{d}}{\mathrm{d} t} \tilde{V}\left(\boldsymbol{x}_{t}(\varphi)\right)+2 \tilde{\beta} \tilde{V}\left(\boldsymbol{x}_{t}(\varphi)\right) \leq 0 .
$$

This inequality brings about the following one 


$$
\tilde{V}\left(\boldsymbol{x}_{t}(\varphi)\right) \leq \mathrm{e}^{-2 \tilde{\beta} t} \tilde{V}(\varphi), \text { for } t \geq 0
$$

Thus,

$$
\tilde{\alpha}_{1}\|\boldsymbol{x}(t, \varphi)\|^{2} \leq \tilde{V}\left(\boldsymbol{x}_{t}(\varphi)\right) \leq \mathrm{e}^{-2 \tilde{\beta} t} \tilde{V}(\varphi) \leq \tilde{\alpha}_{2} \mathrm{e}^{-2 \tilde{\beta} t}\|\varphi\|_{\tau}^{2} \quad \text { for } t \geq 0 .
$$

Then, $\|x(t, \varphi)\| \leq \sqrt{\frac{\tilde{\alpha}_{2}}{\tilde{\alpha}_{1}}} \mathrm{e}^{-2 \tilde{\beta} t}\|\varphi\|_{\tau}, t \geq 0$.

\subsection{The Stabilization of the Multiple Time-Delays System}

Considering the controlled uncertain system with multiple time-delays in the following form

$$
\begin{aligned}
& \dot{\boldsymbol{x}}(t)=(\boldsymbol{A}+\Delta \boldsymbol{A}) \boldsymbol{x}(t)+\sum_{i=1}^{m}\left(\boldsymbol{B}_{i}+\Delta \boldsymbol{B}_{i}\right) \boldsymbol{x}\left(t-\tau_{i}\right)+\boldsymbol{d}(t)+\boldsymbol{D} \boldsymbol{u}(t), \\
& \boldsymbol{x}(\theta)=\varphi(\theta), \quad \theta \in[-\tau, 0],
\end{aligned}
$$

where $\boldsymbol{D} \in \mathbb{R}^{n \times 1}$. We design the state feedback control as $\boldsymbol{u}(t)=\boldsymbol{E x}(t)$ which $\boldsymbol{E} \in \mathbb{R}^{1 \times n}$. For convenience, let $\tilde{\boldsymbol{V}}=\boldsymbol{D E}$.

Theorem 3 The controlled multiple time-delays system (32) is exponential stability if there exist real positive matrices $\tilde{\boldsymbol{P}}, \tilde{\boldsymbol{Q}}_{i},(i=1,2, \cdots, m) \in \mathbb{R}^{n \times n}$ and the constant $\tilde{\beta}>0$ and $\tilde{\rho}>0$ such that the conditions

$$
\begin{aligned}
& \tilde{\mathcal{M}}\left(\tilde{\boldsymbol{P}}, \tilde{\boldsymbol{Q}}_{1}, \tilde{\boldsymbol{Q}}_{2}, \cdots, \tilde{\boldsymbol{Q}}_{m}\right)+\tilde{\rho} \boldsymbol{K}(\omega, \boldsymbol{u})+2 \tilde{\beta} \tilde{\mathcal{M}}(\tilde{\boldsymbol{P}})<0 \\
& \tilde{\boldsymbol{P}}<\tilde{\rho} \boldsymbol{I}
\end{aligned}
$$

hold. Then

$$
\|\boldsymbol{x}(t, \varphi)\| \leq \sqrt{\frac{\tilde{\alpha}_{2}}{\tilde{\alpha}_{1}}} \mathrm{e}^{-\tilde{\beta} t}\|\varphi\|_{\tau}
$$

where

$$
\begin{aligned}
& \tilde{\mathcal{M}}\left(\tilde{\boldsymbol{P}}, \tilde{\boldsymbol{Q}}_{1}, \tilde{\boldsymbol{Q}}_{2}, \cdots, \tilde{\boldsymbol{Q}}_{m}\right) \\
& =\operatorname{diag}\left(\tilde{\boldsymbol{P}} \boldsymbol{A}+\boldsymbol{A}^{\mathrm{T}} \tilde{\boldsymbol{P}}+\sum_{i=1}^{m} \tilde{\boldsymbol{Q}}_{i}+\tilde{\boldsymbol{G}}_{0}+\tilde{\boldsymbol{G}}_{0}^{\mathrm{T}}+\tilde{\boldsymbol{V}}+\tilde{\boldsymbol{V}}^{\mathrm{T}},-\mathrm{e}^{-2 \tilde{\beta} \tau_{1}} \tilde{\boldsymbol{Q}}_{1}, \cdots,-\mathrm{e}^{-2 \tilde{\beta} \tau_{m}} \tilde{\boldsymbol{Q}}_{m}\right) \\
& +\left(\boldsymbol{B}^{\mathrm{T}}+\boldsymbol{G}^{\mathrm{T}}\right) \tilde{\boldsymbol{P}} \boldsymbol{E}_{*}+\boldsymbol{E}_{*}^{\mathrm{T}} \tilde{\boldsymbol{P}}(\boldsymbol{B}+\boldsymbol{G})
\end{aligned}
$$

with

$$
\begin{gathered}
\boldsymbol{B}=\left[\boldsymbol{B}_{1}, \boldsymbol{B}_{2}, \cdots, \boldsymbol{B}_{m}\right], \quad \boldsymbol{G}=\left[\tilde{\boldsymbol{G}}_{1}, \tilde{\boldsymbol{G}}_{2}, \cdots, \tilde{\boldsymbol{G}}_{m}\right], \quad \boldsymbol{E}_{*}=[\boldsymbol{I}_{n}, \underbrace{\mathbf{0}_{n}, \cdots, \mathbf{0}_{n}}_{m-1}], \\
\boldsymbol{E}=\operatorname{diag}[\underbrace{\boldsymbol{I}_{n}, \boldsymbol{I}_{n}, \cdots, \boldsymbol{I}_{n}}_{m}], \tilde{\mathcal{M}}(\tilde{\boldsymbol{P}})=\operatorname{diag}\{\underbrace{\tilde{\boldsymbol{P}}, \underbrace{\mathbf{o}_{n}, \cdots, \mathbf{0}_{n}}_{n}\} .}_{m}
\end{gathered}
$$

And $\tilde{\rho}, \tilde{\beta}, \tilde{\alpha}_{1}, \tilde{\alpha}_{2}, \boldsymbol{K}(\omega, \boldsymbol{u}), \tilde{\mathcal{M}}(\tilde{\boldsymbol{P}})$ are defined in Theorem 2.

Proof. The controlled uncertain system with multiple time-delays (32) can be rewritten as 


$$
\dot{\boldsymbol{x}}(t)=(\boldsymbol{A}+\Delta \boldsymbol{A}+\tilde{\boldsymbol{V}}) \boldsymbol{x}(t)+\sum_{i=1}^{m}\left(\boldsymbol{B}_{i}+\Delta \boldsymbol{B}_{i}\right) \boldsymbol{x}\left(t-\tau_{i}\right)+\boldsymbol{d}(t)
$$

Then, the proof is similar to that of Theorem 2.

Example 2 These matrixes in Theorem 3 are chose when $m=2$ as follows

$$
\begin{gathered}
\tilde{\boldsymbol{P}}=\left[\begin{array}{cc}
0.2 & 0.09 \\
0.09 & 0.1
\end{array}\right], \quad \tilde{\boldsymbol{Q}}_{1}=\left[\begin{array}{cc}
0.4 & 0.9 \\
0.9 & 81
\end{array}\right], \quad \tilde{\boldsymbol{Q}}_{2}=\left[\begin{array}{cc}
0.9 & 0.4 \\
0.4 & 4
\end{array}\right], \\
\boldsymbol{A}=\left[\begin{array}{cc}
-5 & 0.8 \\
0.6 & -1
\end{array}\right], \quad \boldsymbol{B}_{1}=\left[\begin{array}{cc}
0.2 & 0.1 \\
0 & 0.1
\end{array}\right], \quad \boldsymbol{B}_{2}=\left[\begin{array}{cc}
0.1 & 0.8 \\
0 & 0.1
\end{array}\right], \\
\Delta \boldsymbol{A}=\left[\begin{array}{cc}
0.1 \sin (t) & 0.1 \sin (t) \\
0.1 \sin (2 t) & 0.1 \sin (3 t)
\end{array}\right], \Delta \boldsymbol{B}_{1}=\left[\begin{array}{cc}
0.1 \cos (t) & 0.1 \cos (t) \\
0.1 \cos (2 t) & 0.1 \cos (3 t)
\end{array}\right], \\
\Delta \boldsymbol{B}_{2}=\left[\begin{array}{cc}
0.1 \cos (t) & 0.1 \cos (t) \\
0.1 \cos (2 t) & 0.1 \cos (3 t)
\end{array}\right], \quad \tilde{\boldsymbol{G}}_{1}=\tilde{\boldsymbol{G}}_{2}=\tilde{\boldsymbol{G}}_{3}\left[\begin{array}{cc}
0.01 & 0.01 \\
0.01 & 0.01
\end{array}\right], \\
\tilde{\boldsymbol{V}}=\boldsymbol{D} \boldsymbol{E}=\left[\begin{array}{c}
0 \\
19
\end{array}\right] *\left[\begin{array}{ll}
0.02 & -2.7
\end{array}\right] .
\end{gathered}
$$

We choose

$$
\begin{array}{ccc}
\Delta \boldsymbol{A} \leq 0.2, & \Delta \boldsymbol{B}_{1} \leq 0.2, & \Delta \boldsymbol{B}_{2} \leq 0.2, \\
\tau_{1}=0.2, & \tau_{2}=0.5, \quad \tilde{\beta}=0.2 .
\end{array}
$$

By calculating, we obtained

$$
\begin{aligned}
& \tilde{\mathcal{M}}\left(\tilde{\boldsymbol{P}}, \tilde{\boldsymbol{Q}}_{1}, \tilde{\boldsymbol{Q}}_{2}\right)+\tilde{\rho} \boldsymbol{K}(\omega, \boldsymbol{u})+2 \tilde{\beta} \tilde{\mathcal{M}}(\tilde{\boldsymbol{P}}) \\
& =\left[\begin{array}{ccccccc}
-0.3167 & 1.5913 & 0.0429 & 0.0319 & 0.0265 & 0.1764 \\
1.5913 & -17.4207 & 0.0199 & 0.0209 & 0.0149 & 0.0889 \\
0.0429 & 0.0199 & -0.2581 & -0.7997 & 0 & 0 \\
0.0319 & 0.0209 & -0.7997 & -87.5710 & 0 & 0 \\
0.0265 & 0.0149 & 0 & 0 & -0.9240 & -0.3133 \\
0.1764 & 0.0889 & 0 & 0 & -0.3133 & -4.7104
\end{array}\right],
\end{aligned}
$$

which the eigenvalues are

$$
\begin{aligned}
& \lambda_{1}=-87.5783, \quad \lambda_{2}=-17.5680, \quad \lambda_{3}=-4.7432, \\
& \lambda_{4}=-0.8985, \quad \lambda_{5}=-0.2690, \quad \lambda_{6}=-0.1439 .
\end{aligned}
$$

Thus, the matrix $\tilde{\mathcal{M}}\left(\tilde{\boldsymbol{P}}, \tilde{\boldsymbol{Q}}_{1}, \tilde{\boldsymbol{Q}}_{2}\right)+\tilde{\rho} \boldsymbol{K}(\omega, \boldsymbol{u})+2 \tilde{\beta} \tilde{\mathcal{M}}(\tilde{\boldsymbol{P}})$ ia a negative matrix and $\tilde{\boldsymbol{P}}<\tilde{\rho} \mathbf{I}$. The two conditions in Theorem 3 are satisfied. At this time, the solution of the system meets

$$
\|\boldsymbol{x}(t, \varphi)\| \leq 19.8293 \mathrm{e}^{-0.2 t}\|\varphi\|_{\tau} .
$$

When we select the initial value as $\varphi(\theta)=(-2,2)^{\mathrm{T}}, t \in[-3,0]$, the trajectories of the state variables $x_{1}(t)$ and $x_{2}(t)$ for the uncertain system with multiple delays (without the controller) are shown in Figure 3 while the Figure 4 illustrates the trajectories of the state variables $x_{1}(t)$ and $x_{2}(t)$ for the controlled uncertain system with multiple delays (32). Numerical simulation results reveal the effectiveness of our proposed method. 


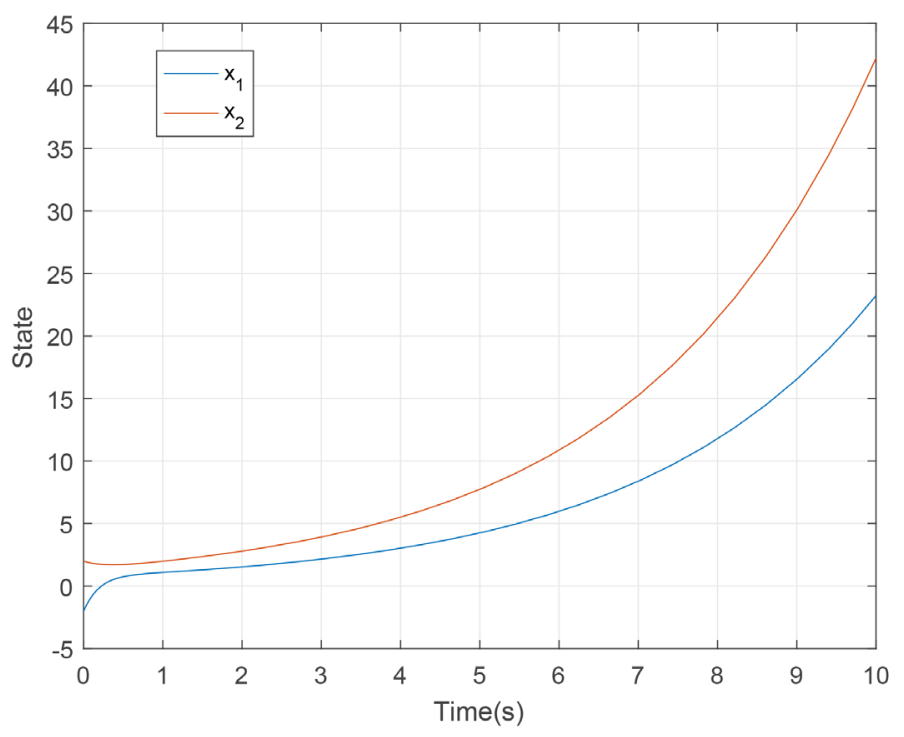

Figure 3. The trajectories of the state variables $x_{1}(t)$ and $x_{2}(t)$ for the uncertain multiple time-delays system without the controller (19).

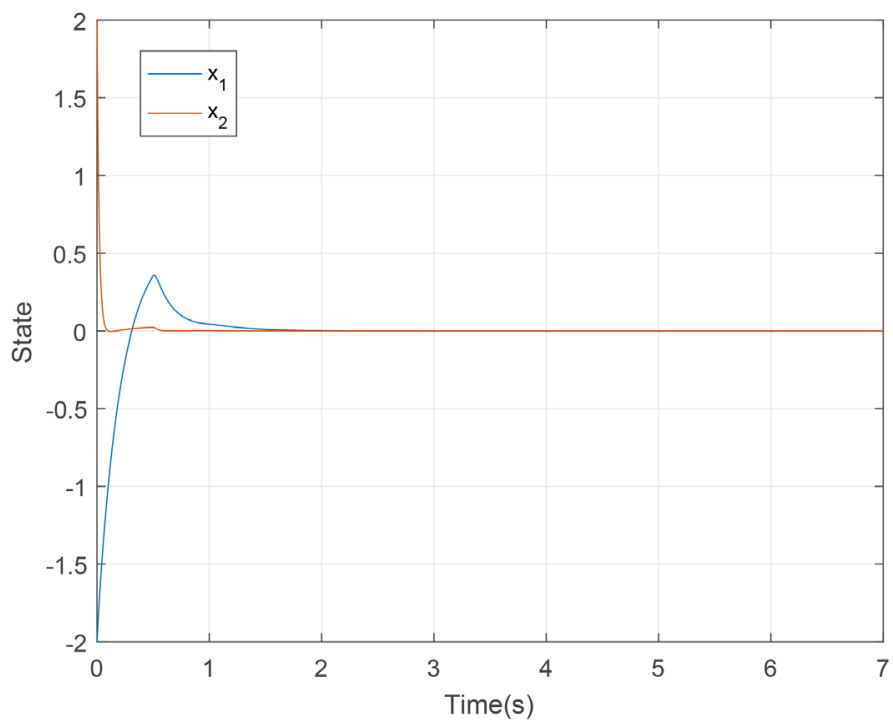

Figure 4. The trajectories of the state variables $x_{1}(t)$ and $x_{2}(t)$ for the controlled uncertain system with multiple time-delays (32).

\section{Conclusion}

In this article, the issue of exponential stability for a class of uncertain linear systems with a single time-delay (or multiple time-delays) is researched. The exponential stability for multiple time-delays linear system under double uncertainties is an innovative point. Firstly, we analyze the stability of the uncertain linear single time-delay systems according to the the Lyapunov-Krasovskii functional. The stability conditions are revealed in the form of the linear matrix inequality (LMI). In order to stabilize the solution of the single time-delay (or multiple time-delays) systems at the equilibrium point, we designed the state 
feedback control. Thus, we give the corresponding stabilization criteria which you can refer to the Theorem 1, 3. Finally, Numerical simulations show that the state variables of the system quickly stabilize to the equilibrium point under the effect of state feedback control $\boldsymbol{u}(t)$. This also shows the effectiveness of the proposed method. In the future, we will consider the stability and stabilization of uncertain nonlinear systems with multiple time-delays.

\section{Acknowledgements}

We appreciate for Editors' and Reviewers' warm work earnestly. Their comments and suggestions are very meaningful to our research work.

\section{Funding}

This work is partly supported by The Postgraduate Innovation Fund Project of Sichuan University of Science and Engineering (Grant No. y2020079).

\section{Authors Contributions}

WQ PAN proposed the main the idea and the proofs of this paper. TZ LI prepared the manuscript initially and gave the numerical simulation of this paper.

\section{Availability of Data and Material}

The data used to support the findings of this study are available from the corresponding author upon request.

\section{Competing Interests}

The authors declare that they have no competing interests.

\section{References}

[1] Mondie, S. and Kharitonov, V.L. (2005) Exponential Estimates for Retarded Time Delay Systems: An LMI Approach. IEEE Transactions on Automatic Control, 50, 268-273. https://doi.org/10.1109/TAC.2004.841916

[2] Haidar, A., Boukas, E.K. and Xu, S. (2009) Exponential Stability and Static Output Feedback Stabilization of Singular Time-Delay Systems with Saturating Actuators. IET Control Theory and Applications, 3, 1293-1305. https://doi.org/10.1049/iet-cta.2008.0212

[3] Fridman, E. and Orlov, Y. (2009) Exponential Stability of Linear Distributed Parameter Systems with Time-Varying Delays. Automatica, 45, 194-201. https://doi.org/10.1016/j.automatica.2008.06.006

[4] Guo, L., Gu, H. and Xing, J. (2012) Asymptotic and Exponential Stability of Uncertain System with Interval Delay. Applied Mathematics and Computation, 218, 9997-10006. https://doi.org/10.1016/j.amc.2012.03.074

[5] Zhang, B., Lam, J. and Xu, S. (2010) Absolute Exponential Stability Criteria for a Class of Nonlinear Time-Delay Systems. Nonlinear Analysis Real World Applications, 11, 1963-1976. https://doi.org/10.1016/j.nonrwa.2009.04.018

[6] Nam, P.T. and Phat, V.U.N. (2008) Robust Exponential Stability and Stabilization of 
Linear Uncertain Polytopic Time-Delay Systems. Journal of Control Theory and Applications, 6, 163-170. https://doi.org/10.1007/s11768-008-6104-4

[7] Chen, Y., Xue, A. and Lu, R. (2008) On Robustly Exponential Stability of Uncertain Neutral Systems with Time-Varying Delays and Nonlinear Perturbations. Nonlinear Analysis, 68, 2464-2470. https://doi.org/10.1016/j.na.2007.01.070

[8] Tian, Y., Cai, Y. and Sun, Y. (2015) Exponential Stabilization of a Class of Time-Varying Delay Systems with Nonlinear Perturbations. Mathematical Problems in Engineering, 2015, Article ID: 737949. https://doi.org/10.1155/2015/737949

[9] Deng, W., Li, C. and Lü, J.H. (2006) Stability Analysis of Linear Fractional Differential System with Multiple Time Delays. Nonlinear Dynamics, 48, 409-416. https://doi.org/10.1007/s11071-006-9094-0

[10] Jiang, Y.L. and Jiang, T. (2014) A Novel LMI Criterion for Power System Stability with Multiple Time-Delays. Science China (Technological Sciences), 57, 1392-1400. https://doi.org/10.1007/s11431-014-5569-2

[11] Wu, F.X. (2016) Stability Analysis of Genetic Regulatory Networks with Multiple Time Delays. 29th Annual International Conference of the IEEE Engineering in Medicine and Biology Society, Lyon, 22-26 August 2007, 1387-1390. https://doi.org/10.1109/IEMBS.2007.4352557

[12] Li, J., Chen, Z. and Cai, D. (2016) Delay-Dependent Stability Control for Power System with Multiple Time-Delays. IEEE Transactions on Power Systems, 31, 2316-2326. https://doi.org/10.1109/TPWRS.2015.2456037

[13] Li, Z.Y., Fan, Q. and Li, L. (2019) Stability Analysis of Linear Continuous-Time Delay-Difference Systems with Multiple Time-Delays. Journal of the Franklin Institute, 356, 11492-11519. https://doi.org/10.1016/j.jfranklin.2019.08.004

[14] Chen, D.L. and Zhang, W.D. (2014) Sliding Mode Control of Uncertain Neutral Stochastic Systems with Multiple Delays. Mathematical Problems in Engineering, 2008, Article ID: 761342. https://doi.org/10.1155/2008/761342

[15] Zhang, H.H. and Xin, J.E. (2015) Robust Stochastic Stability for Uncertain Stochastic System with Multiple Delays. Journal of Shandong Uiversity (Engineering Science), 45, 54-63.

[16] Li, T.Z., Wang, Y. and Xiang, Z.G. (2014) Stability of a Class of Fractional-Order Nonlinear Systems. Discrete Dynamics in Nature and Society, 2014, Article ID: 724270. https://doi.org/10.1155/2014/724270

[17] Wang, Y. and Li, T. (2014) Stability Analysis of Fractional-Order Nonlinear Systems with Delay. Mathematical Problems in Engineering, 2014, Article ID: 301235. https://doi.org/10.1155/2014/301235

[18] Wen, Y., Zhou, X.F. and Zhang, Z. (2015) Lyapunov Method for Nonlinear Fractional Differential Systems with Delay. Nonlinear Dynamics, 82, 1015-1025. https://doi.org/10.1007/s11071-015-2214-y

[19] Podlubny, I. (1999) Fractional Differential Equations. Academic, New York. 